Supporting Information

\title{
Enhancing dry adhesion of polymeric micropatterns by electric fields
}

Vaishali Chopra ${ }^{1,2}$, Maciej Chudak ${ }^{3}$, René Hensel' ${ }^{1}$ Anton A. Darhuber ${ }^{3}$, Eduard Arzt ${ }^{1,2 *}$

${ }^{1}$ INM - Leibniz Institute for New Materials, 66123 Saarbrücken, Germany

2 Department of Materials Science and Engineering, Saarland University, 66123

Saarbrücken, Germany

${ }^{3}$ Department of Applied Physics, Eindhoven University of Technology, 5600 MB Eindhoven, Netherlands

*corresponding author: eduard.arzt@leibniz-inm.de 


\section{Section S1. Dependence of the electroadhesion force on the $a / b$ ratio.}

Figure S1 shows the dependence of the electroadhesion force on $a / b$, i.e. the ratio of the electrode width to the array period.

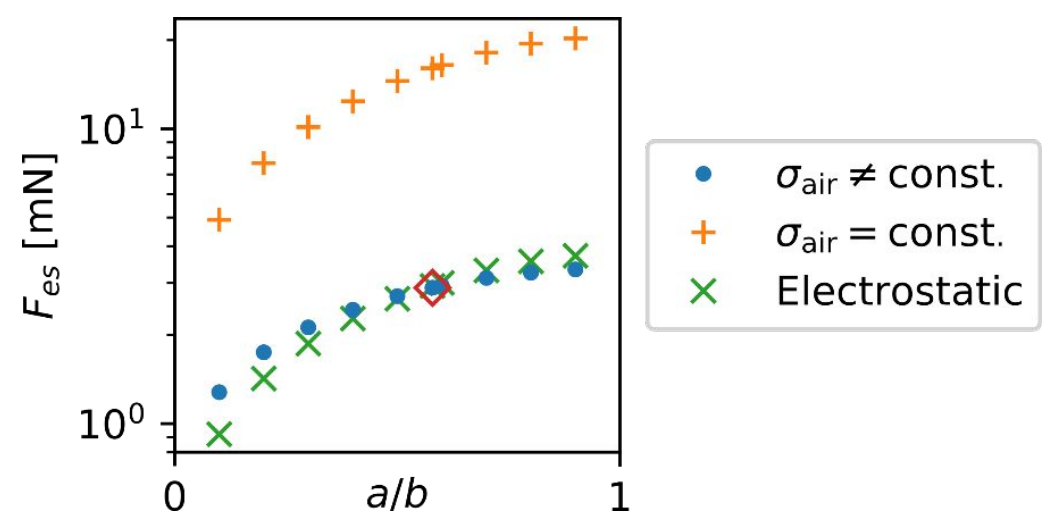

Figure S1. Dependence of the electroadhesion force on the $a / b$ ratio for $\delta=1 \mu \mathrm{m}$ and $V_{0}=2 \mathrm{kV}$. Blue points are for $E_{c}=3 \mathrm{MV} / \mathrm{m}$ and $s_{L}=1 \mu \mathrm{m} / \mathrm{V}$.

\section{Section S2. Model validation for flat surfaces}

For validation we compared our numerical model to the results reported in ref. 1 . Therefore, simulations were performed using the geometry similar to that shown in Fig. S2(c), without a pillar array. Cao et al. report that in a flat-flat contact (i.e., nonpatterned elastomer film and a flat wall as a target surface) the average adhesion stress $\sigma_{a d}$ has the form

$$
\sigma_{a d}=\frac{1}{2} \varepsilon_{0}\left(\frac{V_{0}}{2 b}\right)^{2}\left[\left(\frac{\varepsilon_{w}}{\varepsilon_{0}}\right)^{2}-1\right] C=\sigma_{0} C
$$

where $V_{0}$ is the voltage applied to the electrodes, $b$ is the half-period of the electrodes, $\varepsilon_{w}$ is the permittivity of the wall, $C$ is a dimensionless stress scale function dependent on the geometric parameters of the system, i.e. electrode width, elastomer and air gap thickness as well as the permittivity ratio of the elastomer and wall. We define $\sigma_{0} \equiv \frac{\sigma_{a d}}{C}$. This equation was derived with the approximation that the system is periodic with symmetry planes through the center of each electrode and antisymmetry planes in between the electrodes. 
Figure S3 shows the influence of chosen dielectric and geometric parameters on the electroadhesion stress scale. Our numerical results almost quantitatively reproduce the theoretical prediction based on Eq. (1). They slightly underestimate the stress values, by 5 to $13 \%$. This may be due to the different numerical methods employed, as the cited values were obtained with a point matching method described in Ref. ${ }^{2}$.
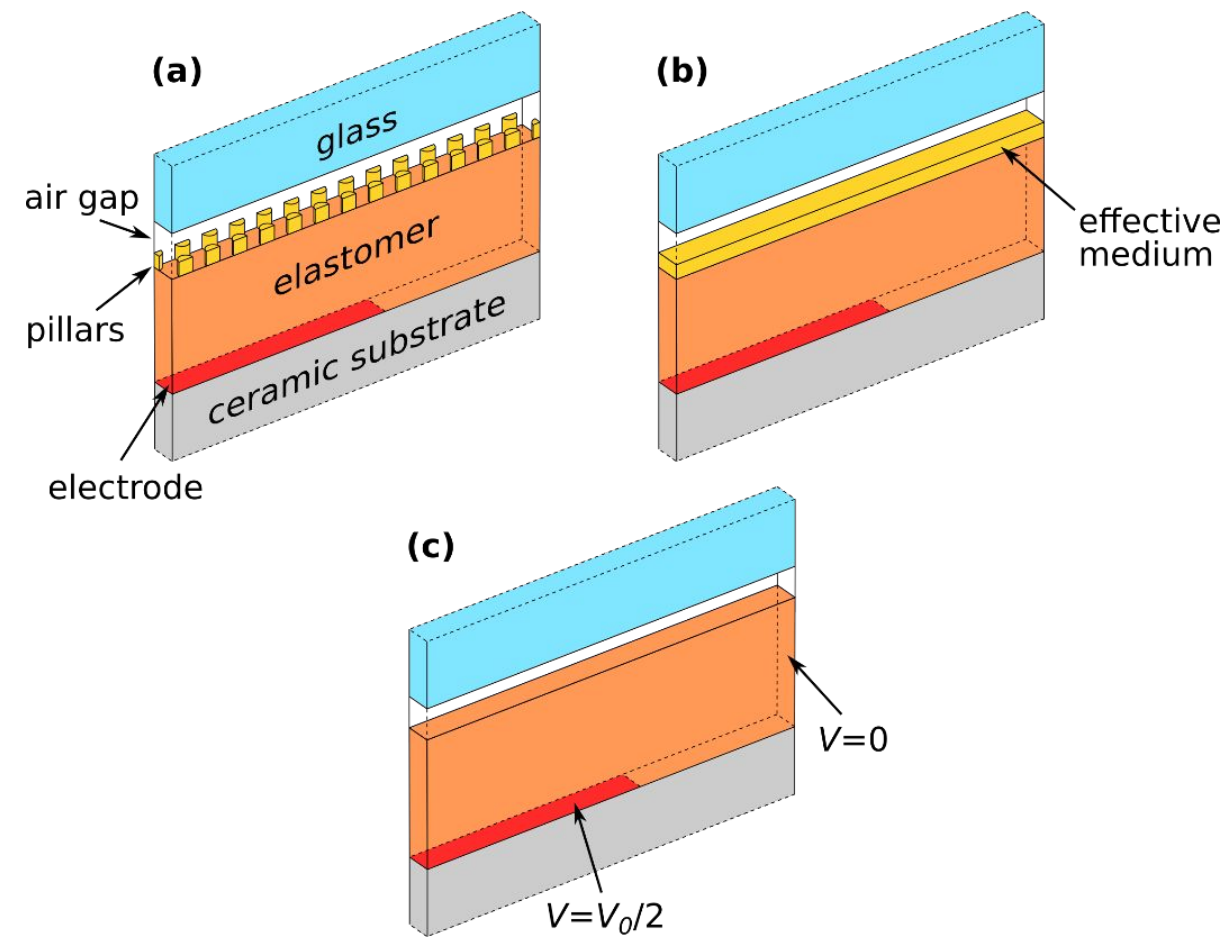

Figure S2. Illustration of the periodic numerical model geometry. (a) Realistic representation of the hexagonal pillar array. (b) Pillar array layer represented by an effective medium approximation. (c) Model with no pillar array or effective medium.

\section{Section S3. Validation of the effective medium approximation}

In order to validate the effective medium representation of the micropillar array, we consider a periodic cell model, assuming an infinite electrode array contacting an infinite, flat object. In this case an elementary unit cell can be defined, bounded by adjacent symmetry planes. Figure $\mathrm{S} 2(\mathrm{a}, \mathrm{b})$ shows two considered geometries, (a) a realistic representation of the hexagonal micropillar array and (b) an effective medium approximation. We consider all external boundaries to be symmetric $(\boldsymbol{n} \cdot \boldsymbol{E}=0)$, with exception of the boundary intersecting the space between electrodes, where an antisymmetry condition holds $(V=0)$. 
Figure S4(a) shows the average electroadhesion stress $\sigma_{a d}$ as a function of air gap thickness $\delta$, considering the realistic pillar array geometry (dashed orange line, Fig. S2(a)) and an effective medium approximation (solid blue line, Fig. S2(b)). Both lines overlap and converge to an exponential function (grey dotted line). In Fig. S4(b) the ratio of the two extracted stresses is plotted as a function of $\delta, \sigma_{e f}$ relating to the effective medium approximation and $\sigma_{p i l}$ to the realistic geometry. At $\delta=1 \mu \mathrm{m}$ the effective medium approximation underestimates the pressure by around $2 \%$ but at $\delta>3 \mu \mathrm{m}$ it overestimates the pressure by less than $1 \%$.
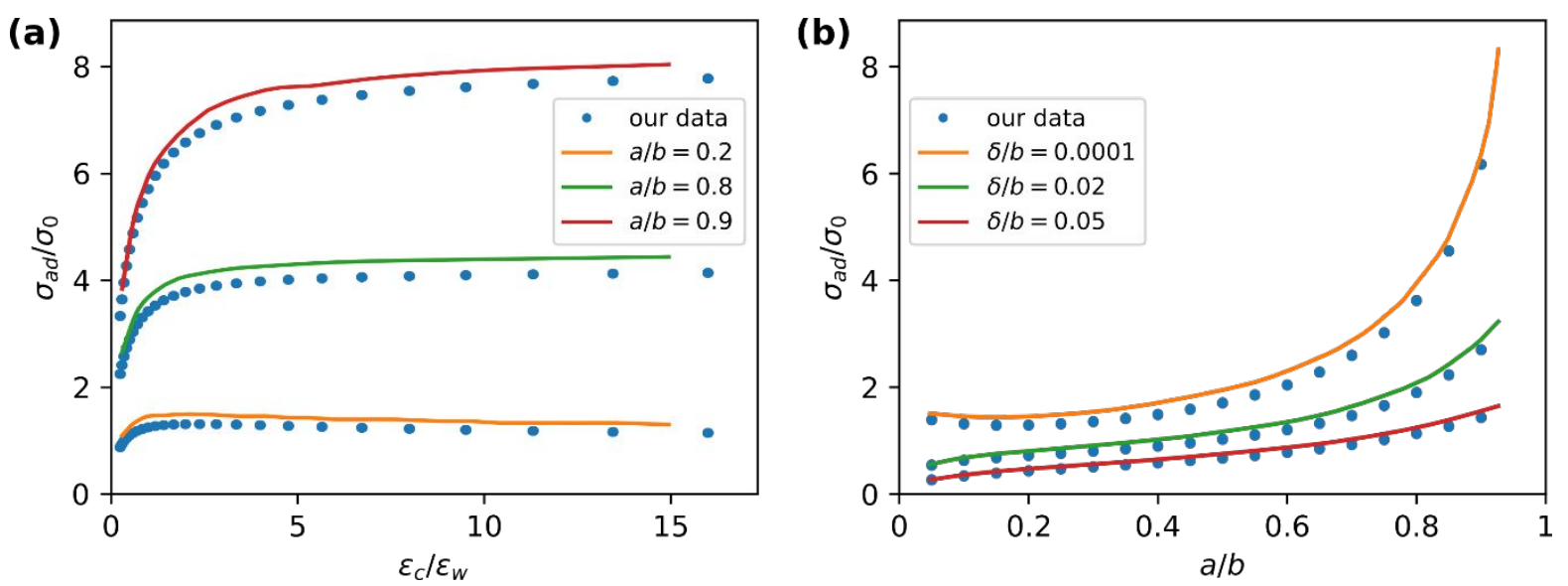

Figure S3. Comparison of the non-dimensional electroadhesion stress $\frac{\sigma_{a d}}{\sigma_{0}}(\mathrm{a})$ as a function of the permittivity ratio $\frac{\varepsilon_{c}}{\varepsilon_{w}}$ of the cover and the wall, respectively, for three values of $\frac{a}{b}$ and (b) as a function of $\frac{a}{b}$ for three values of $\frac{\delta}{b}$. Blue symbols represent our results, solid lines those of ${ }^{1}$.
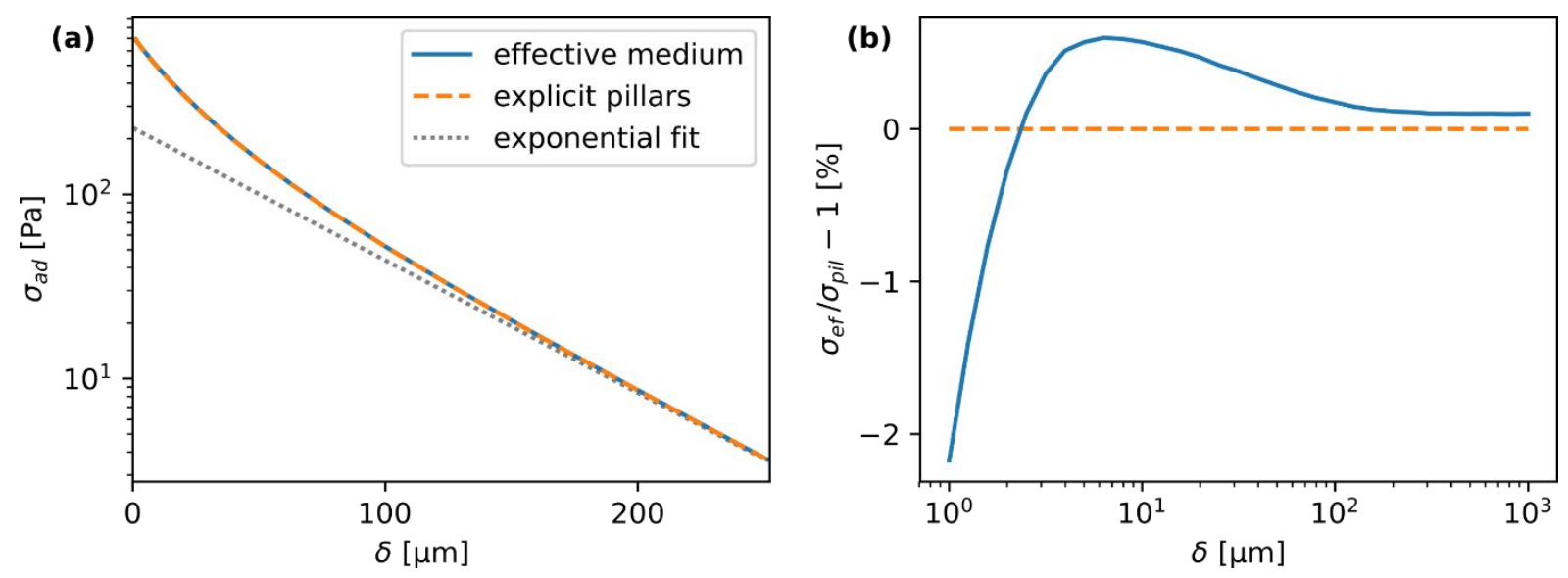
Figure S4. (a) Average electroadhesive pressure considering realistic pillar array geometry (dashed orange lines) and an effective medium approximation (solid blue lines) as a function of air gap thickness $\delta$. Both lines overlap and converge to an exponential function (grey dotted line). (b) Ratio of the two extracted pressures as a function of $\delta$. At $\delta=1 \mu \mathrm{m}$ the effective medium approximation underestimates the pressure by around $2 \%$.

\section{Section S4. Comparison between idealized and realistic geometries of the electrode array}

Figure S5 shows a comparison of logarithmic pseudocolor maps of the electroadhesion pressure for the electrostatic model for $\delta=1 \mu \mathrm{m}$ and $V_{0}=2 \mathrm{kV}$, using different computational domain geometries. (a) A realistic electrode array geometry including horizontal electrode segments, (b) an idealized geometry which assumes a symmetry plane at $y=0$ and an antisymmetry plane at $x=0$ (dashed white lines) and (c) an infinite array, derived from the periodic unit cell model, presented in section S3 of this document. In (c) the pressure map is obtained by tiling the periodic cell results. Here, the curvature of the lens is accounted for by spatially varying the air gap thickness (akin to the Derjaguin approximation known from the theory of van der Waals forces $\left.{ }^{3,4}\right)$. The fringe fields at the edges of the electrode array are noticeably different in each of the three models. This difference, however, accounts for less than $3 \%$ of the total force on the object and is therefore not significant. Model (a) is the most realistic, but also the computationally most expensive. Model (b) reduces the required number of mesh elements by $75 \%$. Model (c) is computationally the most efficient and allows to include the micropillars explicitly, but does not allow to realistically consider electrical conduction and requires approximating the shape of the target if it isn't flat and parallel to the array. We decided to use model (b) in the main text. 

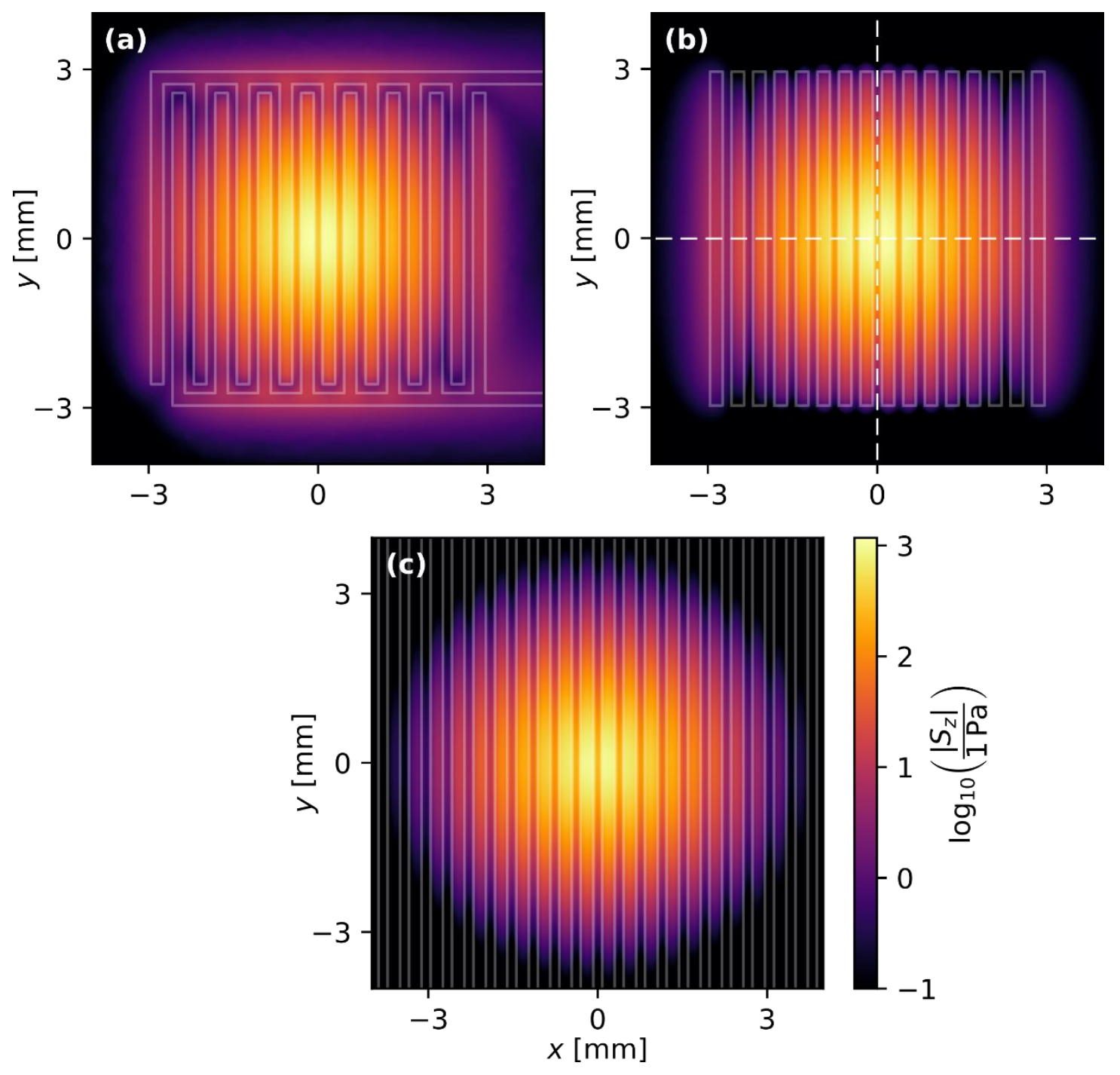

Figure 5. Logarithmic pseudocolor maps of the electroadhesion pressure for different electrode array shapes represented by the faint white lines, when $\delta$ $=1 \mu \mathrm{m}$ and $V_{0}=\mathbf{2} \mathrm{kV}$. (a) Realistic electrode geometry including horizontal electrode segments. (b) Assuming a symmetry plane at $y=0$ and an antisymmetry plane at $x$ $=0$ (dashed white lines). (c) Infinite array, derived from the periodic unit cell model.

\section{Section S5. Scaling of Maxwell traction for purely dielectric materials}

We consider a simplified system of a bilayer of two materials $a$ (air) and $b$ (lens) sandwiched between two parallel plate electrodes, to make it amenable to an analytical treatment. In this case the electric field vector only has a single nonzero Cartesian component. The Maxwell traction acting at the interface scales 


$$
\varepsilon_{a} E_{a}^{2}-\varepsilon_{b} E_{b}^{2}=\varepsilon_{a} E_{a}\left(E_{a}-E_{b}\right)=\varepsilon_{a} E_{a}^{2}\left(1-\frac{\varepsilon_{a}}{\varepsilon_{b}}\right)=\frac{\varepsilon_{b}-1}{\varepsilon_{b}} E_{a}^{2}
$$

where in the first and second steps we have used the discontinuity condition $\varepsilon_{a} E_{a}=\varepsilon_{b}$ $E_{b}$. In the last step, we used $\varepsilon_{\text {air }} \approx 1$. The traction, therefore, approaches a constant for large $\varepsilon_{b}$ (essentially all the voltage drop will be in the $a$ phase and also $E_{a}$ will become constant). Similarly, it scales as $\varepsilon_{b}-1$ for small $\varepsilon_{b} \approx 1$ (because now $E_{a} \approx E_{b}$ both become constants, which are determined by the spacing of the electrodes). Both limits are in accordance with the results in Fig. $7(\mathrm{c})$ in the manuscript.

The electroadhesion geometry includes two air-lens interfaces instead of one. However, the electric field at the more distant interface further away from the electrode array is already so weak due to the rapid exponential decay of the field strength, that its contribution to $F_{e s}$ is negligible.

\section{References}

(1) Cao, C.; Sun, X.; Fang, Y.; Qin, Q. H.; Yu, A.; Feng, X. Q. Theoretical Model and Design of Electroadhesive Pad with Interdigitated Electrodes. Mater. Des. 2016, 89, 485-491. https://doi.org/10.1016/j.matdes.2015.09.162.

(2) Marcuse, D. Electrostatic Field of Coplanar Lines Computed with the Point Matching Method. IEEE J. Quantum Electron. 1989, 25 (5), 939-947. https://doi.org/10.1109/3.27984.

(3) Derjaguin, B. Untersuchungen Über Die Reibung Und Adhäsion, IV. KolloidZeitschrift 1934, 69 (2), 155-164. https://doi.org/10.1007/BF01433225.

(4) Parsegian, V. A. Van Der Waals Forces; Cambridge University Press, 2005. https://doi.org/10.1017/CBO9780511614606. 